\title{
Sub-wavelength InAs quantum dot micro- disk lasers epitaxially grown on exact Si (001) substrates
}

\section{Citation}

Wan, Yating, Qiang Li, Alan Y. Liu, Weng W. Chow, Arthur C. Gossard, John E. Bowers, Evelyn L. $\mathrm{Hu}$, and Kei May Lau. 2016. "Sub-Wavelength InAs Quantum Dot Micro-Disk Lasers Epitaxially Grown on Exact Si (001) Substrates." Applied Physics Letters 108 (22) (May 30): 221101. doi:10.1063/1.4952600.

\section{Published Version}

doi:10.1063/1.4952600

\section{Permanent link}

http://nrs.harvard.edu/urn-3:HUL.InstRepos:33983359

\section{Terms of Use}

This article was downloaded from Harvard University's DASH repository, and is made available under the terms and conditions applicable to Other Posted Material, as set forth at http:// nrs.harvard.edu/urn-3:HUL.InstRepos:dash.current.terms-of-use\#LAA

\section{Share Your Story}

The Harvard community has made this article openly available.

Please share how this access benefits you. Submit a story.

Accessibility 


\title{
Sub-wavelength InAs quantum dot micro-disk lasers epitaxially grown on exact $\mathrm{Si}(001)$ substrates
}

\author{
Yating Wan, ${ }^{1, a)}$ Qiang Li, ${ }^{1, a)}$ Alan Y. Liu, ${ }^{2, a)}$ Weng W. Chow, ${ }^{3}$ Arthur C. Gossard, ${ }^{2,4}$ \\ John E. Bowers, ${ }^{2,4}$ Evelyn L. Hu, ${ }^{5}$ and Kei May Lau ${ }^{1, b)}$ \\ ${ }^{1}$ Department of Electronic and Computer Engineering, Hong Kong University of Science and Technology, \\ Clear Water Bay, Kowloon, Hong Kong \\ ${ }^{2}$ Materials Department, University of California Santa Barbara, Santa Barbara, California 93106, USA \\ ${ }^{3}$ Sandia National Laboratories, Albuquerque, New Mexico 87185-1086, USA \\ ${ }^{4}$ Department of Electrical and Computer Engineering, University of California Santa Barbara, Santa Barbara, \\ California 93106, USA \\ ${ }^{5}$ School of Engineering and Applied Sciences, Harvard University, Cambridge, Massachusetts 02138, USA
}

(Received 15 February 2016; accepted 19 April 2016; published online 31 May 2016)

\begin{abstract}
Subwavelength micro-disk lasers (MDLs) as small as $1 \mu \mathrm{m}$ in diameter on exact (001) silicon were fabricated using colloidal lithography. The micro-cavity gain medium incorporating five-stacked InAs quantum dot layers was grown on a high crystalline quality GaAs-on-V-grooved-Si template with no absorptive intermediate buffers. Under continuous-wave optical pumping, the MDLs on silicon exhibit lasing in the $1.2-\mu \mathrm{m}$ wavelength range with low thresholds down to $35 \mu \mathrm{W}$ at $10 \mathrm{~K}$. The MDLs compare favorably with devices fabricated on native GaAs substrates and state-of-theart work reported elsewhere. Feasibility of device miniaturization can be projected by sizedependent lasing characteristics. The results show a promising path towards dense integration of photonic components on the mainstream complementary metal-oxide-semiconductor platform. Published by AIP Publishing. [http://dx.doi.org/10.1063/1.4952600]
\end{abstract}

In the quest for integration with mature miniaturized electronics, there is growing interest in developing efficient, compact, and integrable laser sources on the well-established complementary metal-oxide-semiconductor (CMOS) platform. ${ }^{1}$ The whispering-gallery mode (WGM) micro-disk laser (MDL) is considered as an attractive light source for on-chip optical communications, data-processing, and chemical sensing applications. ${ }^{2}$ Proof-of-principle demonstration of a lowfootprint optical interconnect by heterogeneous integration of micro-disk lasers and micro-detectors has been reported. ${ }^{3}$ Shrinking of the micro-disk diameter is desirable for dense integration and low power consumption. However, there exist challenges associated with higher radiation loss, limited gain medium, and surface recombination in small volume laser cavities. ${ }^{45}$ To minimize the recombination on the resonator surface and achieve ultra-low threshold lasing in small volume cavities, the use of quantum dots (QDs) instead of quantum wells as the active media is being actively investigated. ${ }^{4,6-8}$ Recently, Song et al. ${ }^{6}$ have reported single-mode lasing in $0.7-\mu \mathrm{m}$ diameter quantum dot micro-disk lasers on GaAs in the wavelength range of $860-880 \mathrm{~nm}$ under pulsed excitation at $10 \mathrm{~K}$. Kryzhanovskaya et al. ${ }^{4}$ have demonstrated room temperature continuous-wave $(\mathrm{CW})$ lasing in $1-\mu \mathrm{m}$ micro-disk quantum dot lasers on GaAs in the 1.29-1.32- $\mu$ m wavelength range. However, at sub-wavelength cavity sizes (diameter of the disk $\mathrm{D}<\lambda$ ) scale, high radiation loss remains a serious issue. As a result, the above-mentioned examples represent the rather limited results on native GaAs substrates reported in the literature. So far, no sub-wavelength micro-disk lasers on Si have been reported, either by bonding or by direct epitaxy.

\footnotetext{
${ }^{\text {a) }}$. Wan, Q. Li, and A. Y. Liu contributed equally to this work.

b)Electronic mail: eekmlau@ust.hk. Tel: (852)23587049, Fax: (852) 23581485.
}

In this work, we report low-threshold lasing in subwavelength QD micro-disk lasers on exact-oriented $\mathrm{Si}(001)$ substrates under $\mathrm{CW}$ optical excitation. The active laser structures were grown on a GaAs-on-V-grooved-Si (GoVS) template $^{9}$ that is free of antiphase-domains and absorptive intermediate buffers. Compared to the quantum dot lasers recently demonstrated on offcut silicon using Ge buffers, ${ }^{10-14}$ or direct nucleation of GaAs, ${ }^{15,16}$ the use of on-axis (001) silicon offers better compatibility with conventional $\mathrm{Si}$ CMOS processes. The promising lasing characteristics of the MDLs also suggest a viable route towards large-scale, lowcost integration with passive optical components on a silicon-on-insulator (SOI) platform.

Fig. 1(a) presents a cross-sectional transmission electron microscope (TEM) image of the entire micro-disk laser structure grown on silicon. We first grew a $1 \mu \mathrm{m}$ coalesced GaAs layer on a V-groove-patterned $\mathrm{Si}$ (001) substrate by metalorganic chemical vapor deposition (MOCVD). ${ }^{9,17,18}$ This was followed by the deposition of a $1 \mu \mathrm{m}$ GaAs buffer, a $600 \mathrm{~nm}$ $\mathrm{Al}_{0.7} \mathrm{Ga}_{0.3} \mathrm{As}$ post region, and a $500 \mathrm{~nm}$ disk region in a molecular beam epitaxy (MBE) system. ${ }^{19}$ The disk region encapsulates five layers of InAs/InGaAs dot-in-a-well (DWELL) structure. A schematic of the epitaxial layers in the disk region is shown in Fig. 1(b). Fig. 1(c) is a zoomed-in TEM image of the five-stacked QD active region, presenting the uniform dot arrays. The good crystalline quality of the active device region is attributed to an effective defect trapping and reduction scheme through the V-grooved structure at the $\mathrm{GaAs} / \mathrm{Si}$ hetero-interface, as revealed by the zoomed-in TEM image in Fig. 1(d). Fig. 1(e) presents the normalized photoluminescence (PL) spectra at room temperature from the as-grown QDs under increasing excitation power from 1.65 to $330 \mu \mathrm{W}$. Ground state emission in the $1.3 \mu \mathrm{m}$ telecommunication 
a

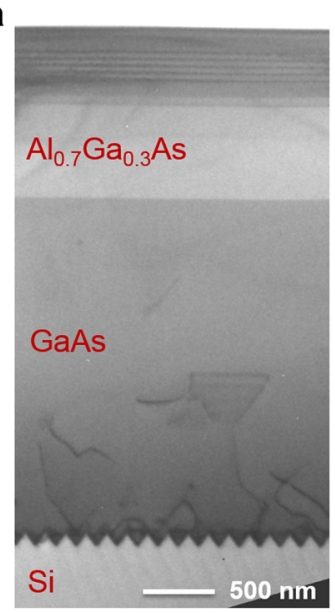

b

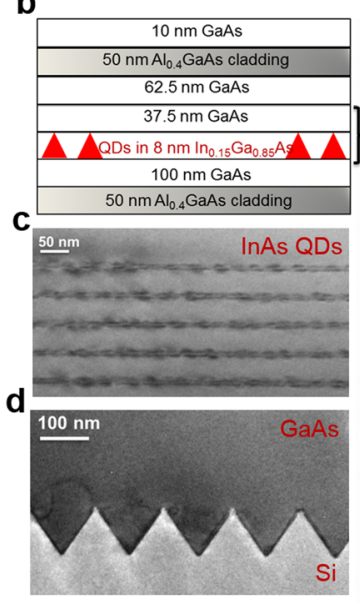

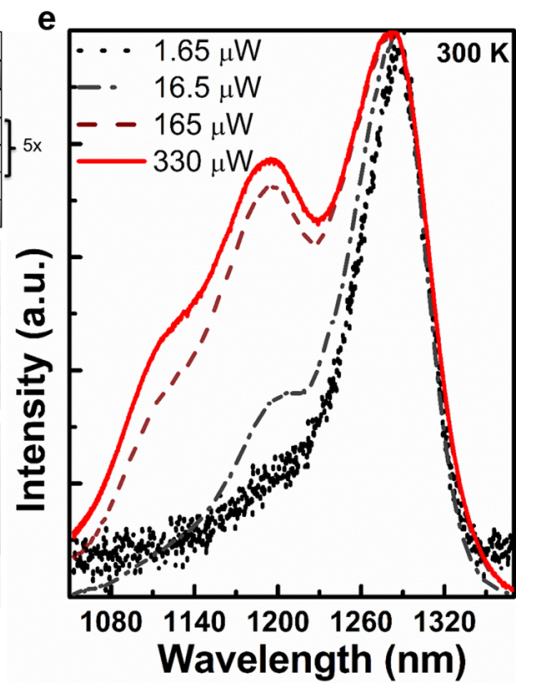

FIG. 1. (a) Cross-sectional TEM image of the micro-disk structure grown on the GoVS substrate; (b) schematic illustration of the epitaxial structure of material in the disk region; (c) highresolution TEM image of the five-stack InAs QDs; (d) cross-sectional TEM image of the V-grooved structure, showing defect trapping and localization; (e) room temperature photoluminescence spectrum of the as grown structure at progressively higher excitations. wavelength band was observed. A shoulder appearing on the high energy side with increased pump power suggests emission from excited states. The wide inhomogeneous linewidth $(\sim 20 \mathrm{meV})^{20}$ allows for easy coupling into the resonant modes from small volume cavities with a large free spectral range (FSR). ${ }^{21}$

We used low cost colloidal lithography to fabricate ultra-small micro-disks. Silica colloidal beads $(1 \mu \mathrm{m}$ in diameter) were dispersed on the sample as hard masks. Inductively coupled plasma (ICP) etching was performed to transfer the pattern $(\sim 1.1 \mu \mathrm{m})$ onto the full structure. The etching parameters have been optimized to achieve nonselective and isotropic etching to form vertical and smooth sidewalls. The chamber pressure was maintained at 5 mTorr, with the coil and platen power set as $500 \mathrm{~W}$ and $100 \mathrm{~W}$, respectively. $\mathrm{BCl}_{3}$ and $\mathrm{Ar}$ mixed gases were used with flow rates of $20 \mathrm{sccm}$ and $6.5 \mathrm{sccm}$, respectively. We found that the substrate temperature has a strong effect on the etch rate, selectivity, profile, and surface roughness. The chamber temperature was thus set at $10^{\circ} \mathrm{C}$, with thermally conductive glue applied on the back of the wafers to provide good thermal conductance between the chuck and the wafer. After 70s etching, the remaining silica beads were removed in acetone in an ultrasonic bath. Finally, the $200 \mathrm{~nm} \mathrm{Al}_{0.7} \mathrm{Ga}_{0.3} \mathrm{As}$ sacrificial layer was etched laterally from the outer periphery using dilute hydrofluoric acid (HF) to form a supporting pedestal. Fig. 2(a) presents a $70^{\circ}$ tilted scanning electron microscope (SEM) image of a fabricated disk. The top-down view of the disk in Fig. 2(b) shows the circular geometry. A zoomed-in view of a $90^{\circ}$ tilted image in Fig. 2(c) reveals its smooth sidewall.

The sample was mounted in a Helium gas flow cryostat and cooled to $10 \mathrm{~K}$ for micro-photoluminescence ( $\mu \mathrm{PL})$ measurements in a surface-normal pump/collection configuration. Individual MDLs were excited by a $\mathrm{CW}$ diode laser operating at $532 \mathrm{~nm}$. The exciting light was focused to a spot size of approximately $4 \mu \mathrm{m}$ in diameter and approximately $50 \%$ of the power reaching the sample surface was absorbed. ${ }^{22}$ The pump intensity was varied using polarizers. The emitted light was collected from the top of the microdisks and dispersed through a monochromator with a $500 \mathrm{~mm}$ focal length before reaching a liquid-nitrogencooled InGaAs detector array. Fig. 3(a) presents a set of emission spectra collected at various pump intensities, offset vertically for clarity. A weak cavity mode accompanied by a broad background emission appears at a low pump intensity of $8.25 \mu \mathrm{W}$. Once surpassing the threshold, the dominant peak increases sharply in intensity. The small diameter of the micro-cavities leads to a large free spectral range (FSR) of $\Delta \lambda \approx \frac{\lambda^{2}}{2 \pi R n_{\text {eff }}}=\frac{[1.2 \mu]^{2}}{2 \pi(0.5 \mu) 3.46}=132 \mathrm{~nm},{ }^{23}$ wherein $\mathrm{n}_{\mathrm{eff}}$ is the effective refractive index of the cavity, $\mathrm{R}$ is the disk radius, and $\lambda$ represents the emission wavelength. The large FSR results in well-separated neighboring resonances and likelihood of single mode operation. Fig. 3(b) plots the light-out/ light-in (LL) curve. The output power exhibits a superlinear increase with pump power, along with a pronounced narrowing of mode linewidth (Fig. 3(c)), a clear sign of lasing. The a

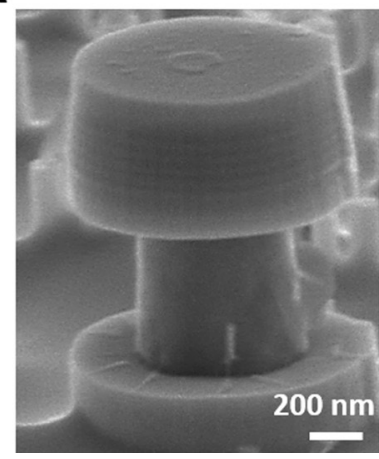

b

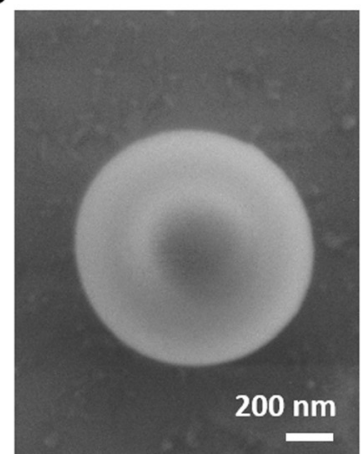

C

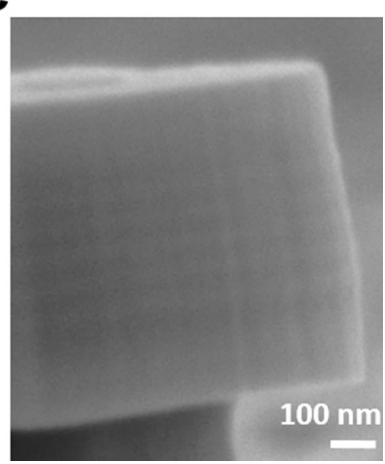

FIG. 2. (a) $70^{\circ}$ tilted SEM image of the fabricated micro-disk; (b) top-view image of the disk showing its circularity; (c) a zoomed-in view of the sidewall in a $90^{\circ}$ tilted SEM image, revealing its smoothness and vertical profile. 

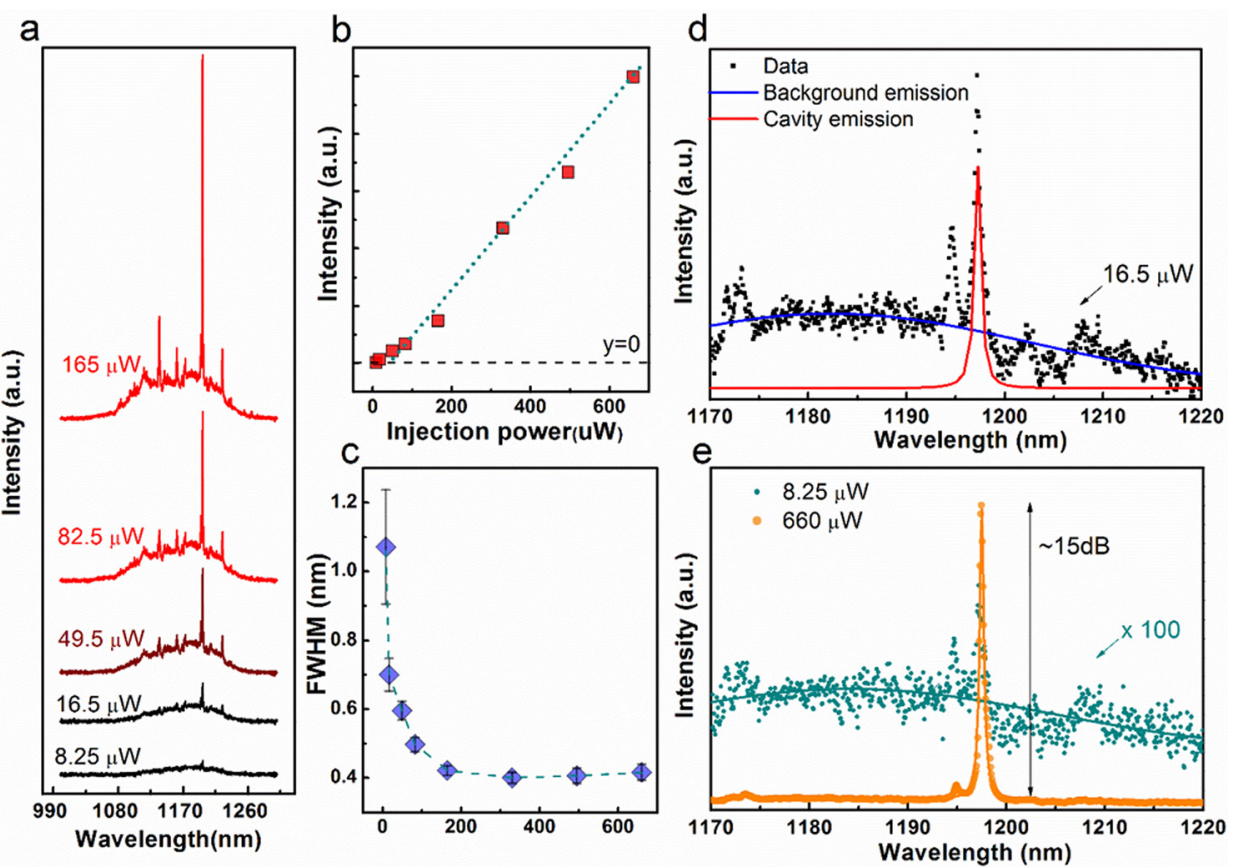

FIG. 3. (a) Power dependent PL spectra, presenting the transition from spontaneous emission to lasing; (b) L-L curve. The kink indicates the onset of superlinear emission and lasing operation. The blue dashed line represents a fit to the experimental data and the black dashed line represents the position where intensity equals to zero. (c) Mode linewidth as a function of pump intensity. It is noted that the fitting error of the linewidth at low pump intensity is too large to determine the real value. (d) PL spectrum below the threshold $(16.5 \mu \mathrm{W})$. The symbols represent the measured data, the blue line is a fit to the broad InAs QDs photoluminescence spectrum background, and the red line is a fit to the narrow cavity emission. (e) Laser emission spectra measured below (light blue, $8.25 \mu \mathrm{W}$ ) and above (yellow, $660 \mu \mathrm{W}$ ) the threshold. Symbols are measured data and lines are Gaussian fits. The spectrum below the threshold (light blue, $8.25 \mu \mathrm{W})$ was amplified 100 times to be visible.

lasing threshold is extrapolated to be as low as $35 \mu \mathrm{W}$ from the LL curve. Using the linewidth at a pump power of $16.5 \mu \mathrm{W}$, somewhat below the threshold, we estimate a cold cavity Q-factor of $\sim 1720$, as depicted in Fig. 3(d). To denote the optical transition positions in the gain medium, emission below threshold $(8.25 \mu \mathrm{W})$ was amplified by 100 times and overlaid with the emission well above threshold $(660 \mu \mathrm{W})$, shown in Fig. 3(e). A high extinction ratio of $\sim 15 \mathrm{~dB}$ was observed.

To identify the lasing modes visible in the disk spectra, we numerically calculated the resonant modes using the finite-difference time-domain (FDTD) method. Here, we only consider transverse electric (TE)-polarized modes with first radial mode number and different azimuthal mode number in the simulations. The $\mathrm{TE}_{1,5}$ mode (radial mode number $l=1$, azimuthal mode number $m=5$ ) has a resonant wavelength at $1.194 \mu \mathrm{m}$, which matches well with the measured lasing wavelength at $1.197 \mu \mathrm{m}$. The obtained top view and cross-sectional view of the electric field distribution of the $\mathrm{TE}_{1,5}$ resonance are presented in Figs. 4(a) and 4(b), respectively, showing the field is mostly concentrated at the disk periphery. Simulation also shows that with smaller cavity size, the evanescent field at the disk periphery couples more effectively to the external environment, leading to greater radiative loss. Although these results suggest that the coupling of laser output may be advantageous for smaller cavities, the sharply diminishing quality factor $Q$ with further decrease of the radius of the micro-disks is also an important consideration.

The spontaneous emission factor $(\beta)$ of the lasing mode was extracted by fitting the experimental data to a semiconductor cavity-QED model. ${ }^{24}$ In the model, equations of motion for carrier and photon populations, as well as photon correlations, are solved numerically for a given carrier injection rate into the quantum-dot states. Input to the model is the light-matter coupling coefficient and the dephasing rate. The former is computed from the active region and optical resonator configurations and is represented by a Rabi frequency of $6 \times 10^{10} \mathrm{~s}^{-1}$. For the dephasing rate, we assume a value of $1 \mathrm{ps}^{-1}$ for $10 \mathrm{~K}$ and lasing carrier densities. We use the onset of linewidth reduction with increasing pump intensity from the experiment to help determine where to expect the bends in the "S" shape of the input/output intensity loglog plot. Fig. 4(c) plots the computed input/output curves for the values of $\beta$ that bound the experimental data. The fitted results are further confirmed by the agreement between calculation and experiment for the linewidth versus pump intensity shown in Fig. 4(d). The best fit to the experimental data gives a spontaneous emission factor $\beta$ of 0.3 .

Micro-disk cavities were simultaneously fabricated on a GaAs substrate for comparison with those on the GaAs-onV-grooved-Si substrate. A statistical distribution of the lasing threshold and central wavelength is overlaid with the normalized broad PL background in Fig. 4(e). The lasing wavelengths are narrowly distributed near the PL peak wavelengths at $10 \mathrm{~K}$. A large ratio of quality factor over mode volume $Q / V_{\text {eff }} \approx 8600 \mu \mathrm{m}^{-3}$ guarantees the large spontaneous emission rate ${ }^{25}$ and gives rise to low threshold lasing. The threshold for the micro-disk lasers on silicon ranges from 35 to $67 \mu \mathrm{W}$, with an average value of $50 \mu \mathrm{W}$, approximately 1.5 times the average value for lasers on the GaAs substrate $(33 \mu \mathrm{W})$. As the small diameter disks have limited area of gain on the ground state transitions, a sufficiently high modal 

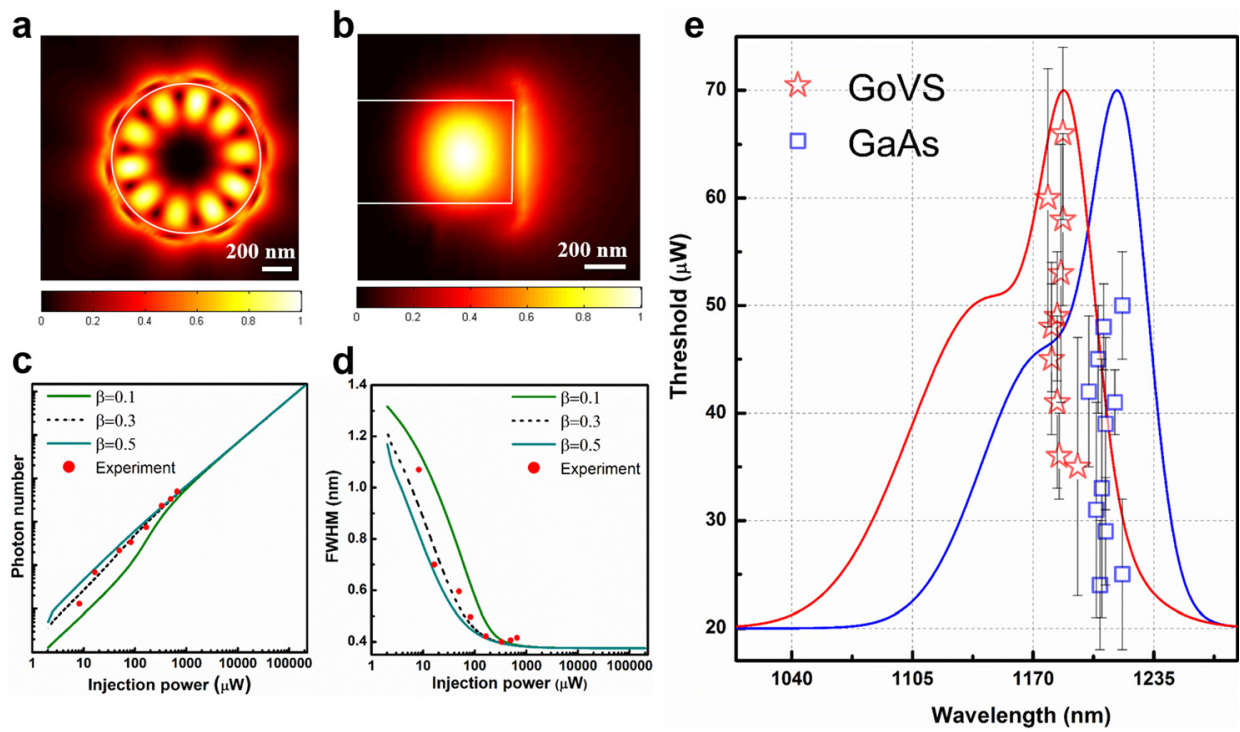

FIG. 4. Top view (a) and crosssectional view (b) of the simulated electric field distribution of the $\mathrm{TE}_{1,5}$ resonance; white and black colors refer to the maximum and minimum of the field density, respectively. The white outline corresponds to the structure boundary. (c) Calculation and experiment for input/output intensity log-log plot. (d) Calculation and experiment for the linewidth versus pump intensity. The best fit to the experimental data gives a spontaneous emission factor $\beta$ of 0.3 . The fits to $\beta$ of $0.1,0.5$ are also presented for comparison. (e) Statistical analysis of the threshold power for micro-disks fabricated on $\mathrm{Si}$ (red stars) and GaAs (black squares); the error bars result from the linear fit to the LL curve. gain from the active medium is essential to balance loss and bring about lasing action. The somewhat larger lasing thresholds on silicon are presumably related to crystalline defects (on the order of $1 \times 10^{8} \mathrm{~cm}^{-2}$ by plan-view transmission electron microscopy) leading to reduced QD modal gain. Moreover, the deep levels associated with the dislocations can also leach away the photogenerated carriers that are generated outside the active region (since the pump laser is of high enough energy that it is absorbed by all layers), so it will effectively reduce the "injection efficiency" or the fraction of carriers entering the active region compared to the GaAs case for the same pump power and result in higher absolute thresholds. The surface-normal pump/collection configuration used limits the coupling of the pump source, as well as the collection efficiency from the MDL. We therefore expect to observe lower thresholds with an optical-fiberbased evanescent coupling technique. ${ }^{26}$

We also fabricated micro-disks with larger diameters of 4 and $5 \mu \mathrm{m}$. Representative LL curves are presented in the supplementary material. ${ }^{27}$ Figs. 5(a) and 5(b) plot the MDL a

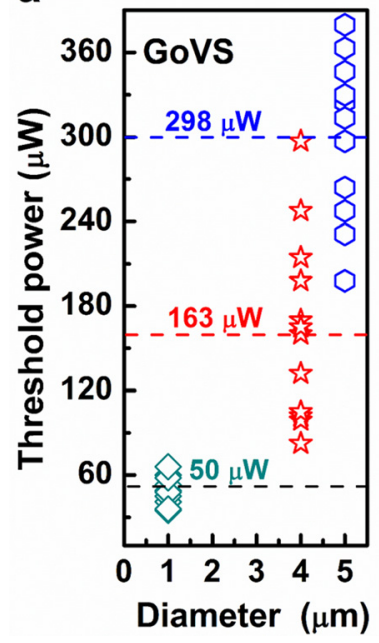

b

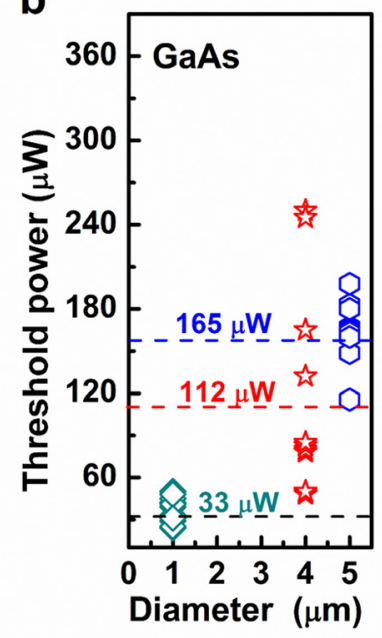

FIG. 5. Threshold power as a function of disk diameters on the GoVS template (a) and the GaAs substrate (b). The dashed lines represent the average threshold. threshold power as a function of disk diameters on the GoVS template and the GaAs substrate. Around ten working lasers were measured for each cavity size. The dashed lines represent the average lasing thresholds. Despite the wide spread of the data, there is a consistent trend of higher thresholds for larger disks. Larger diameter disks possess a smaller free spectral range and thus smaller mode separation. Depending on the distribution of QDs and their overlap with the modes of the cavity, both single mode lasing and multimode lasing were observed for most $4 \mu \mathrm{m}$ and $5 \mu \mathrm{m}$ micro-disks. Due to the mode competition, micro-disk lasers with multi-mode lasing generally show larger thresholds. In addition, the larger disks have larger volumes of material to pump; in particular, the central region of the microdisk absorbs pump power but has no spatial overlap with the WGMs. Understandably, there is a more pronounced dispersion of the thresholds of the dominant modes in larger diameter disks. Nevertheless, we observed an apparent monotonic decrease of threshold power as the disk diameter is scaled from 5 to $1 \mu \mathrm{m}$ on both $\mathrm{GaAs}$ and Si. Compared with the recently reported subwavelength InAs QDs micro-disk lasers on GaAs substrates under pulsed pumping, ${ }^{6}$ our micro-disks on silicon under $\mathrm{CW}$ operation exhibited even lower lasing thresholds at the same working temperature of $10 \mathrm{~K}$ and the same ratio of $\mathrm{d} / \lambda=0.83$ ( $\mathrm{d}$ is the diameter of the disk and $\lambda$ is the lasing wavelength). We expect that higher performance can be achieved after increasing the quality and density of QDs in the active region. ${ }^{20}$

In conclusion, we demonstrated continuous-wave lasing in sub-wavelength InAs quantum-dot micro-disk lasers epitaxially grown on industrial-compatible $\mathrm{Si}$ (001) substrates. By combining the small mode volume and zero dimensional electron density of states of quantum dot micro-disk structures, a low threshold of $35 \mu \mathrm{W}$ was achieved on Si substrates. We believe that the ultra-low threshold and small footprint configurations provide significant insights and inspire future possibilities to incorporate efficient and compact laser sources on a CMOS-compatible platform.

This work was supported in part by Grants (Nos. 614813 and 16212115) from the Research Grants Council of Hong Kong, DARPA (MTO EPHI), and the American 
Institute for Manufacturing (AIM) Integrated Photonics, and the U.S. Department of Energy NNSA Contract No. DEAC04-94AL85000. The authors would like to thank SUNY Poly for providing the initial nano-patterned Si substrates, Wuhan National Laboratory for Optoelectronics (WNLO) for providing facilities to perform optical measurements, and NFF and MCPF of HKUST for technical support. Helpful discussions with C. Zeng, Y. Geng, and B. Shi are also acknowledged.

${ }^{1}$ M. K. Smit, J. van der Tol, and M. T. Hill, Laser Photonics Rev. 6, 1-13 (2012).

${ }^{2}$ M. T. Hill and M. C. Gather, Nat. Photonics 8(12), 908-918 (2014).

${ }^{3}$ J. V. Campenhout, P. R. A. Binetti, P. R. Romeo, P. Regreny, C. Seassal, X. J. M. Leijtens, T. de Vries, Y. S. Oei, R. P. J. van Veldhoven, R. Nötzel, L. D. Cioccio, J. Fedeli, M. K. Smit, D. V. Thourhout, and R. Baets, IEEE Photonics Technol. Lett. 21, 522-524 (2009).

${ }^{4}$ N. V. Kryzhanovskaya, A. E. Zhukov, M. V. Maximov, E. I. Moiseev, I. I. Shostak, A. M. Nadtochiy, Y. V. Kudashova, A. A. Lipovskii, M. M. Kulagina, and S. I. Troshkov, IEEE J. Sel. Top. Quantum Electron. 21, 709-713 (2015).

${ }^{5}$ L. A. Coldren, S. W. Corzine, and M. L. Mashanovitch, Diode LASERS and Photonic Integrated Circuits (Wiley, 2012).

${ }^{6}$ Q. Song, H. Cao, S. T. Ho, and G. S. Solomon, Appl. Phys. Lett. 94, 061109 (2009).

${ }^{7}$ J. Y. Hsing, T. E. Tzeng, K. Y. Chuanga, T. S. Laya, M. Y. Kuob, Y. Y. Tsaib, K. S. Hsub, and M. H. Shih, J. Cryst. Growth 323(1), 457-459 (2011).

${ }^{8}$ P. Michler, A. Kiraz, L. Zhang, C. Becher, E. Hu, and A. Imamoglu, Appl. Phys. Lett. 77, 184 (2000).

${ }^{9}$ Q. Li, K. W. Ng, and K. M. Lau, Appl. Phys. Lett. 106, 072105 (2015).

${ }^{10}$ H. Liu, T. Wang, Q. Jiang, R. Hogg, F. Tutu, F. Pozzi, and A. Seeds, Nat. Photonics 5, 416-419 (2011).
${ }^{11}$ A. Lee, Q. Jiang, M. Tang, A. Seeds, and H. Liu, Opt. Express 20(20), 22181-22187 (2012).

${ }^{12}$ A. Y. Liu, C. Zhang, J. Norman, A. Snyder, D. Lubyshev, J. M. Fastenau, A. W. K. Liu, A. C. Gossard, and J. E. Bowers, Appl. Phys. Lett. 104, 041104 (2014).

${ }^{13}$ A. Y. Liu, R. W. Herrick, O. Ueda, P. M. Petroff, A. C. Gossard, and J. E. Bowers, IEEE J. Sel. Top. Quantum Electron. 21(6), 1900708 (2015).

${ }^{14}$ A. Y. Liu, S. Srinivasan, J. Norman, A. C. Gossard, and J. E. Bowers, Photonics Res. 3(5), B1-B9 (2015).

${ }^{15}$ T. Wang, H. Liu, A. Lee, F. Pozzi, and A. Seeds, Opt. Express 19(12), 11381-11386 (2011).

${ }^{16}$ S. M. Chen, M. C. Tang, J. Wu, Q. Jiang, V. G. Dorogan, M. Benamara, Y. I. Mazur, G. J. Salamo, A. J. Seeds, and H. Liu, Electron. Lett. 50(20), 1467-1468 (2014).

${ }^{17}$ Y. Wan, Q. Li, Y. Geng, B. Shi, and K. M. Lau, Appl. Phys. Lett 107, 081106 (2015).

${ }^{18}$ Y. Wan, Q. Li, A. Y. Liu, A. C. Gossard, J. E. Bowers, E. L. Hu, and K. M. Lau, Opt. Lett. 41(7), 1664 (2016).

${ }^{19}$ A. Y. Liu, C. Zhang, A. Snyder, D. Lubyshev, J. M. Fastenau, A. W. K. Liu, A. C. Gossard, and J. E. Bowers, J. Vac. Sci. Technol., B 32(2), 02C108 (2014).

${ }^{20}$ W. W. Chow, A. Y. Liu, A. C. Gossard, and J. E. Bowers, Appl. Phys. Lett. 107, 171106 (2015).

${ }^{21}$ N. C. Frateschi and A. F. J. Levi, Appl. Phys. Lett. 66, 2932 (1995).

${ }^{22}$ T. Ide, T. Baba, J. Tatebayashi, S. Iwamoto, T. Nakaoka, and Y. Arakawa, Opt. Express 13(5), 1615-1620 (2005).

${ }^{23}$ A. C. Tamboli, E. D. Haberer, R. Sharma, K. H. Lee, S. Nakamura, and E. L. Hu, Nat. Photonics 1, 61-64 (2007).

${ }^{24}$ C. Gies, J. Wiersig, M. Lorke, and F. Jahnke, Phys. Rev. A 75, 013803-11 (2007).

${ }^{25}$ Y. Ye, Z. J. Wong, X. Lu, X. Ni, H. Zhu, X. Chen, Y. Wang, and X. Zhang, Nat. Photonics 9(11), 733-737 (2015).

${ }^{26}$ K. Srinivasan, M. Borselli, T. J. Johnson, P. E. Barclay, O. Painter, A. Stintz, and S. Krishna, Appl. Phys. Lett. 86, 151106 (2005).

${ }^{27}$ See supplementary material at http://dx.doi.org/10.1063/1.4952600 for representative LL curves of microdisk lasers with varied diameters. 